\title{
Inelastic cross section measurements performed with the CMS experiment
}

\author{
Manfred Jeitler ${ }^{* \dagger}$ \\ Institute of High Energy Physics of the Austrian Academy of Sciences, Vienna, Austria \\ E-mail: manfred.jeitlerecern.ch
}

\begin{abstract}
The first measurement of the cross section of inelastic proton-lead collisions at $5.02 \mathrm{TeV}$ centerof-mass energy per nucleon-pair is presented. The cross section is corrected for effects beyond the experimental acceptance of CMS. Photon-induced interactions are studied in detail and are excluded from the result, as well as quasi-elastic excitation of the lead nuclei. The data is compared to measurements at much lower center-of-mass energies as well as to predictions of event generators and models. Also the inelastic cross section measured in pp collisions at different center-of-mass energies is reported.
\end{abstract}

The European Physical Society Conference on High Energy Physics

22-29 July 2015

Vienna, Austria

\footnotetext{
* Speaker.

${ }^{\dagger}$ For the CMS collaboration.
} 


\section{Introduction}

Total inelastic proton-proton and proton-nucleon cross sections are the most inclusive observable that can be measured in hadronic collisions. They are, among other things, an essential input to the Monte-Carlo models used for modelling hadronic and nuclear collisions (such as PYTHIA [1] for proton-proton and HIJING [2] for heavy-ion collisions) and in particular for the event generators used by cosmic air-shower experiments to model the shower development in the atmosphere (such as EPOS [3] and QGSJettII [4]). Accurate calculations have turned out to be extremely difficult so that measurements at the high energies available at the LHC are very important. While most of the time the LHC produces proton-proton collisions there are dedicated running periods where collisions of heavy ions with each other or collisions of heavy ions with protons are studied.

In the case of collisions of high-intensity proton or ion beams the presence of pileup (several individual hadron collisions within one "bunch crossing" or collision of two beam particle bunches) influences the cross section measurement and has to be corrected for. Therefore the data of the commissioning phase of the LHC when luminosities and pileup were still low are of particular value for such measurements.

\section{Measurement of the inelastic proton-lead (p-Pb) cross section at $\sqrt{s_{N N}}=5.02 \mathrm{TeV}$}

Theoretical models use the so-called Glauber approach [5] to calculate cross sections of nuclear interactions. An additional effect to take into consideration beyond the basic framework are nucleon-nucleon correlations in the nucleus, which lead to fewer central and more peripheral collisions, resulting in an increase of the nuclear cross sections ("anti-screening"). Another important effect is the contribution of diffraction in multiple scatterings where an excited nucleon falls back to the ground state before the end of the scattering, which reduces the overall cross section and is called "inelastic screening". These effects roughly cancel at low energies and LHC measurements are important to check if this also applies at higher energies.

\subsection{Measurement principle}

Measurements have been carried out at a nucleon-nucleon center-of-mass energy of $5.02 \mathrm{TeV}$ using the CMS hadronic forward calorimeter ("HF"), which covers a range in pseudorapidity of $3<|\eta|<5$ (for a detailed description of the CMS detector see [6]). The calorimeter consists of quartz fibers in a steel matrix, which transport the Cherenkov light produced by the charged component of showers to photodetector tubes. The calorimeter is segmented into cells or "towers" of $0.175 \times 0.175$ in azimuth $\phi$ and pseudorapidity $\eta$. The signal with the highest energy from a single tower was used for the analysis.

Data were taken with the proton beam in clockwise and counter-clockwise direction ("leadproton" and "proton-lead" collisions). Luminosities were measured by dedicated van-derMeer scans for each of the two directions. The integrated luminosity recorded by CMS was $(20.7 \pm 0.7) \mathrm{nb}^{-1}$ and $(14.0 \pm 0.5) \mathrm{nb}^{-1}$ for proton-lead and lead-proton collisions, respectively. For this measurement, data were taken when particle bunches passing through the CMS apparatus were recorded by the beam pick-up detectors ("BPTX"), independent of any signals in the CMS detector (so-called "zero-bias trigger"). 
For the cross section measurement the number of observed inelastic events was corrected for acceptance effects by Monte-Carlo simulations to yield the total number of inelastic collisions $N_{\text {inel }}$. The cross section was then obtained by normalising to the integrated luminosity $\mathscr{L}$ using the relation $\sigma_{\text {inel }}=N_{\text {inel }} / \mathscr{L}$.

\subsection{Event selection}

In this analysis two different event selections were used: one of them requires a coincidence of both sides of the detector (double arm) while the other one accepts also events with signals in only one side of the detector (single arm). While the double-arm selection produces cleaner events, the single-arm selection yields higher statistics. The better noise suppression of the double-arm selection allows to apply lower energy thresholds and the coincidence requirement also efficiently removes photo-nuclear events. On the other hand, a fraction of single-diffractive events is also lost in the double-arm selection. Thus, both selections have different sensitivities to contributions from photon-induced $(\gamma-p)$ and hadronic diffractive interactions and are therefore complementary with regard to systematics.

Defining the highest energy in an HF tower on the positive and negative rapidity side as $E_{H F+}$ and $E_{H F-}$, respectively, and $E_{H F}$ as the maximum of these values in the single-arm selection and as their minimum for the double-arm selection, we plot signal, backgrounds and simulation results for this variable and the two selections in Fig. 1.
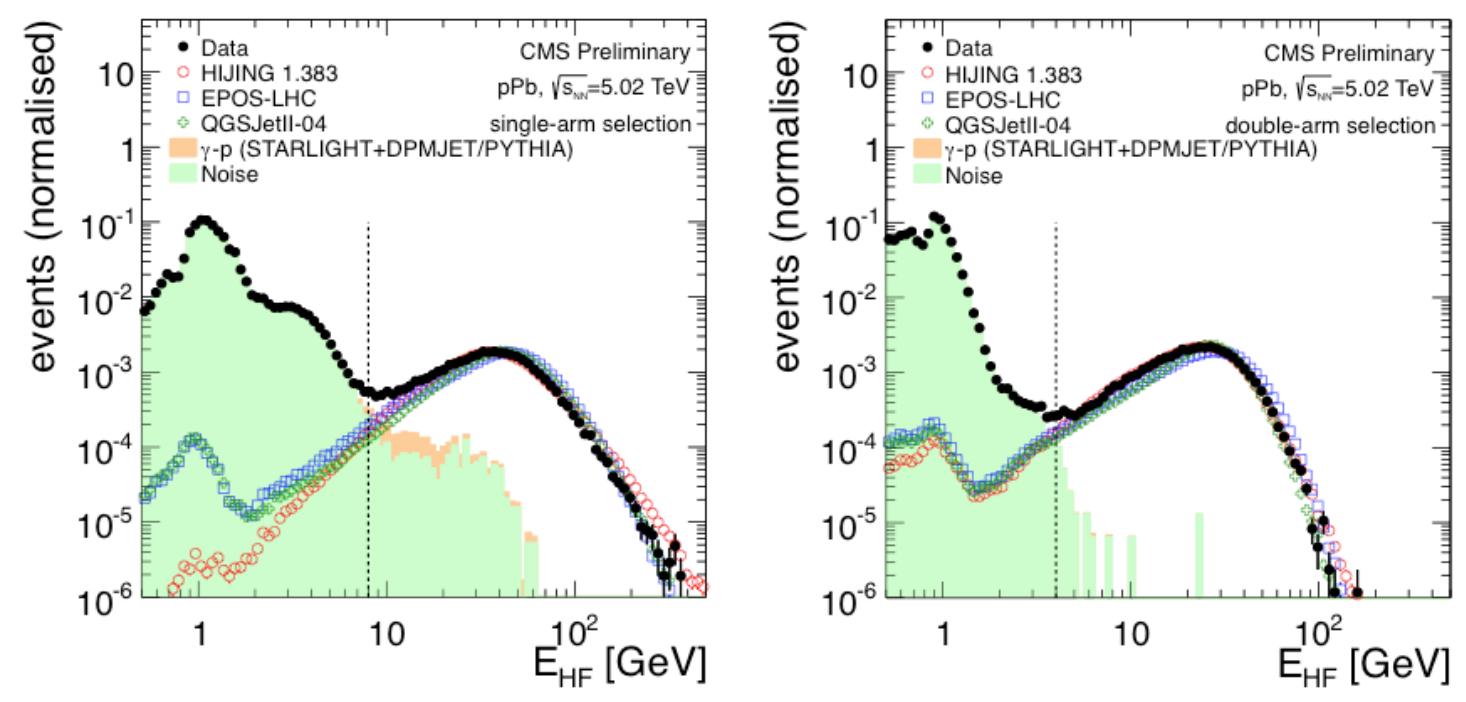

Figure 1: $E_{H F}$ distribution for zero-bias (bunch crossing) triggers (black dots). The noise rate (obtained by issuing random triggers in empty bunch crossings, green) is matched to the zero-bias data. Monte-Carlo simulations of hadronic and electromagnetic processes are matched to the data at high energies $(>10 \mathrm{GeV})$ where background is small. The dotted vertical lines show the energy thresholds used in the analysis.

\subsection{Corrections and uncertainties}

The noise rate and photo-production contribution drop sharply with increasing energy threshold whereas the efficiency drop for the signal is moderate (see Fig. 2). The acceptance correction was calculated from the average of EPOS-LHC and QGSJETII-04 as 
- $\varepsilon_{a c c}=0.939 \pm 0.005$ for the single-arm selection and an energy threshold of $E_{H F}>8 \mathrm{GeV}$ and as

- $\varepsilon_{a c c}=0.910 \pm 0.014$ for the double-arm selection and an energy threshold of $E_{H F}>4 \mathrm{GeV}$.
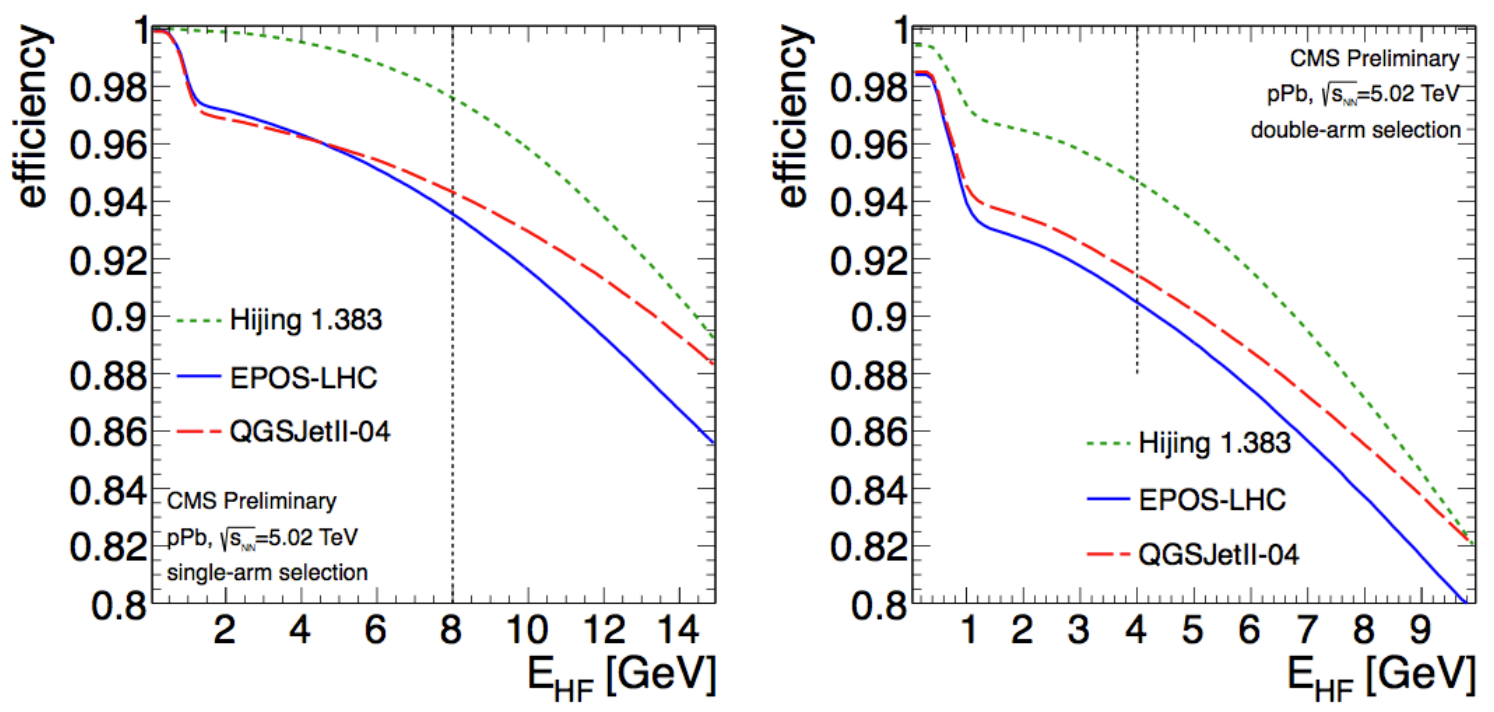

Figure 2: Simulated efficiency against $E_{H F}$ threshold for three different generators. Left: single-arm selection, right: double-arm selection. The dotted vertical lines show the energy thresholds used in the analysis.

Electronic noise and energy depositions from beam gas events have been measured by random triggers issued at times with no beam collisions. The fraction of no-beam events to pass the selection criteria has been determined as $(2.0 \pm 0.5) \times 10^{-3}$ for the single-arm selection with a threshold of $8 \mathrm{GeV}$ and as $(1.8 \pm 0.8) \times 10^{-4}$ for the double-arm selection with a threshold of $4 \mathrm{GeV}$.

Pileup events where several collisions happen within one bunch crossing will at most be counted as one collision event by the detector and therefore have to be corrected for with a factor depending on the instantaneous luminosity, which decreases over the time of an LHC fill because beam particles are being used up. By assuming a Poisson distribution for the number of particle collisions per bunch crossing and iteratively using the inelastic cross section determined in this analysis $\left(\sigma_{\text {inel }}=(2.061 \pm 0.029)\right.$ barn $)$, we arrive for a typical LHC fill at pileup correction factors of 1.025 (start of fill, single-arm), 1.024 (start of fill, double-arm), and 1.011 (end of fill, both selections).

Electromagnetic inelastic particle production in ultra-peripheral ion collisions have been modelled by the STARLIGHT event generator [7]. After applying the HF energy cuts described above still some photo-hadronic events remain, in particular in the single-arm selection. The electromagnetic contribution to the inelastic cross section calculated from the simulation is $(63 \pm 11) \mathrm{mb}$ for the single-arm selection $\left(E_{H F}>8 \mathrm{GeV}\right)$ and $(0.33 \pm 0.05) \mathrm{mb}$ for the double-arm selection $\left(E_{H F}>4 \mathrm{GeV}\right)$. Subtracting these contributions introduces corrections of $3.35 \%$ and $0.02 \%$ for the single-arm and double-arm selection, respectively.

Table 1 summarizes the corrections to be applied to the measurement within the CMS acceptance. 
Table 1: Corrections

\begin{tabular}{|l|l|l|}
\hline & single-arm & double-arm \\
\hline noise & $5.4 \%$ & $0.5 \%$ \\
\hline$\gamma-p$ & $3.4 \%$ & $0.02 \%$ \\
\hline pileup & $1.8 \%$ & $1.8 \%$ \\
\hline phase-space extrapolation & $6 \%$ & $9 \%$ \\
\hline
\end{tabular}

The uncertainty in this measurement is dominated by the determination of the luminosity, which introduces an uncertainty of $2.4 \%$ independent of the event selection method and is correlated for both selections. The other sources of systematic uncertainties are different for the singlearm and the double-arm selection and largely uncorrelated. In both cases they add up to another $2.7 \%$. Adding them in quadrature yields an uncertainty of $4.4 \%$ for each of the two selections and the combination of both selections results in an overall uncertainty of $4.0 \%$.

\subsection{Results}

The cross section was computed as the weighted average over all sub-periods of the data taking period ("runs" subdivided into "luminosity sections" of 23.31 seconds length). The standard deviation of the weighted mean introduces a negligible statistical uncertainty of 0.003 barn. No dependence of the result on the beam direction (ion beam clockwise or counter-clockwise) was observed, which confirms the robustness of the measurement with respect to this parameter. The values obtained for the "visible" cross section (raw value corrected for noise and pileup), its hadronic component (after correcting for photon-proton interactions) and the final value after extrapolation to the full phase space are given in Table 2. The good agreement of the final values for the two selections after all corrections indicates that the corrections are well understood.

Table 2: Results

\begin{tabular}{|l|l|l|}
\hline$\sigma$ (barns) & single-arm $(\mathrm{EHF}>8 \mathrm{GeV})$ & double-arm $(\mathrm{EHF}>4 \mathrm{GeV})$ \\
\hline all selected events & 2.003 & 1.873 \\
\hline hadronic $(\gamma-p$ subtracted $)$ & 1.938 & 1.873 \\
\hline phase-space extrapolation & 2.063 & 2.059 \\
\hline
\end{tabular}

Combining the values for the two selections yields the average result:

$$
\left.\left.\sigma_{\text {inel }}=(2.061 \pm 0.003 \text { (stat. }) \pm 0.039 \text { (syst. }\right) \pm 0.072 \text { (luminosity) }\right) \text { barn }
$$

This is compatible with calculations based on the Glauber theory and suggests that the screening and anti-screening effects described above roughly cancel also for $\mathrm{pPb}$ collisions at $5.02 \mathrm{TeV}$. A more detailed description of this analysis is given in [8]. 


\section{Measurement of diffractive dissociation cross sections in proton-proton collisions at $\sqrt{s}=\mathbf{7} \mathrm{TeV}$}

A significant fraction (approximately 25\%) of the total inelastic pp cross section at high energies is due to diffractive interactions. These are characterized by a large rapidity gap (a range in pseudorapidity $\eta$ or polar angle $\theta$ containing no particles). Diffractive processes in hadronic interactions are considered to be mediated by Pomeron exchange, i.e. the exchange of a color singlet with the quantum numbers of the vacuum. The various types of diffractive as opposed to non-diffractive processes are defined in Fig. 3.

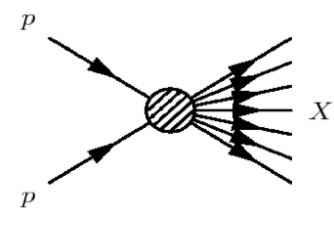

non-diffractive

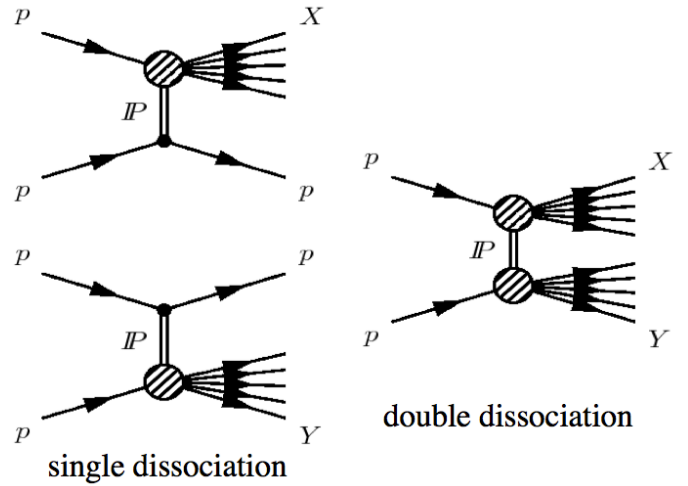

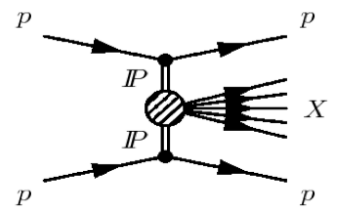

central diffraction

Figure 3: Types of proton collision processes: non-diffractive (ND) vs. diffractive processes: single dissociation (SD), double dissociation (DD) and central diffraction (CD). $X$ and $Y$ denote dissociated protons or centrally produced hadronic systems, with masses $M_{X}$ and $M_{Y}$ calculated from all the four-vectors in the respective group of particles, while $\mathbb{P}$ designates a Pomeron.

Inclusive diffractive cross sections cannot be calculated by perturbative QCD. They are described by Regge theory models, which cannot be extrapolated all the way to LHC energies. The knowledge of these cross sections is crucial for the proper modeling of the full final state of hadronic interactions in Monte-Carlo event generators. The detection of the rapidity gap requires a low probability of overlapping $p p$ interactions in the same bunch crossing (pileup). Therefore, the commissioning phase of the LHC in 2010 was the ideal time to perform such measurements.

In addition to the Forward Calorimeter (HF) described above the very forward region at the negative rapidity end (between -6.6 and -5.2) of CMS was equipped with the CASTOR calorimeter [9] for this measurement. This was instrumental for distinguishing single-dissociation from double-dissociation events (see Fig. 4). The data samples using information from CASTOR $\left(16.2 \mu \mathrm{b}^{-1}\right)$ were taken at a pileup of 0.14 . An alternative analysis based on central pseudorapiditygap events, independent of CASTOR data, was based on $20.3 \mu \mathrm{b}^{-1}$ taken at a negligible pileup of 0.006 .

The trigger was based on the CMS beam scintillator counters (BSC) in conjunction with the BPTX beam pick-up detectors described above. The BSC detectors consisting of 16 scintillator tiles each were located symmetrically at a distance of $\pm 10.86 \mathrm{~m}$ from the CMS interaction point. They covered a pseudorapidity range of $3.23<|\eta|<4.65$ and had an efficiency of $96.3 \%$ for minimum-ionizing particles. 


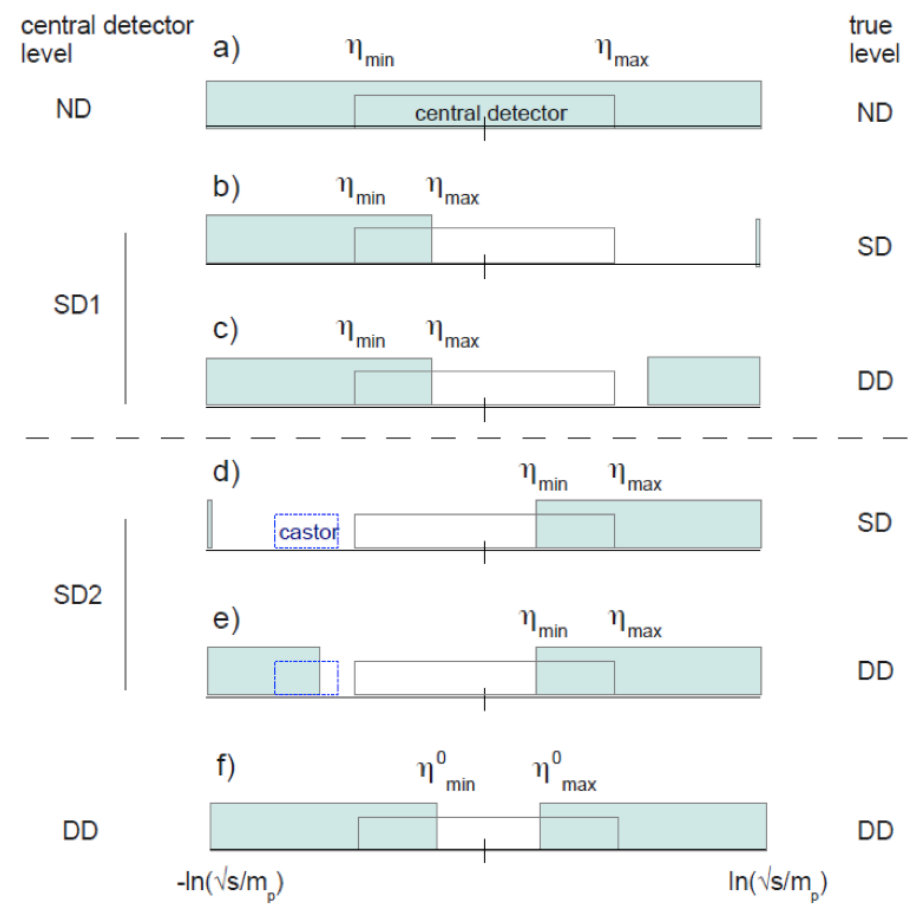

Figure 4: Event topologies of different processes along pseudorapidity $\eta$. The central box denotes the $\eta$ coverage of the CMS central detector. Figures (d) and (e) illustrate how the CASTOR calorimeter allowed to discriminate between single-dissociation events (d) and double-dissociation events where the secondary particles on the negative- $\eta$ side are outside the acceptance of the central detector.

Monte-Carlo simulations allowed to correct the measured distributions for geometrical acceptance and reconstruction efficiency in the detector. Integrated cross sections were extracted using PYTHIA 8 MBR (minimum bias Rockefeller, [10]).

The masses of the two final-state hadronic systems separated by the largest rapidity gap in the event are denoted as $M_{X}$ for the positive- $\eta$ and as $M_{Y}$ for the negative- $\eta$ side. Differential cross sections were measured as functions of $\xi_{X}=M_{X}^{2} / s$ in the region $-5.5<\log _{10} \xi_{X}<-2.5$ for $\log _{10} M_{Y}$ $<0.5$, which is dominated by single dissociation, and $0.5<\log _{10} M_{Y}<1.1$, which is dominated by double dissociation. The CASTOR detector located at the negative-pseudorapidity side allowed to discriminate between the two regions. The inclusive $p p$ cross section was also measured as a function of the width of the central pseudorapidity gap $\Delta \eta$ for $\Delta \eta>3$ with $\log _{10} M_{X}>1.1$ and $\log _{10} M_{Y}>1.1$. This region is dominated by double-dissociation processes. The cross sections in these three regions are well described by the PYTHIA 8 MBR simulations.

The measurements are in agreement with similar measurements performed by the ATLAS collaboration [11]. They are consistent with a slow rise in energy for single-dissociation and doubledissociation cross sections and provide new constraints for the modelling of diffractive processes in hadron collisions. The details of this analysis and the obtained results for the cross sections have been published in [12]. 


\section{References}

[1] T. Sjostrand, S. Mrenna, and P. Z. Skands, "A Brief Introduction to PYTHIA 8.1”, Comput. Phys. Commun. 178 (2008) 852, doi:10.1016/j.cpc.2008.01.036, arXiv:0710.3820.

[2] X.-N. Wang and M. Gyulassy, "HIJING: A Monte Carlo model for multiple jet production in p p, p A and A A collisions”, Phys. Rev. D 44 (1991) 3501, doi:10.1103/PhysRevD.44.3501.

[3] K. Werner, F.-M. Liu, and T. Pierog, "Parton ladder splitting and the rapidity dependence of transverse momentum spectra in deuteron-gold collisions at RHIC”, Phys. Rev. C 74 (2006) 044902, doi:10.1103/PhysRevC.74.044902, arXiv:hep-ph/0506232.

[4] S. Ostapchenko, "Air shower calculations with QGSJET-II: Effects of Pomeron loops", Nucl. Phys. Proc. Suppl. 196 (2009) 90, doi:10.1016/j.nuclphysbps.2009.09.014.

[5] R.J. Glauber, Nucl.Phys. B 21 (1970) 135 and Phys.Rev. 100 (1955) 242)

[6] CMS Collaboration, "The CMS experiment at the CERN LHC”, JINST 03 (2008) S08004.

[7] A. J. Baltz, Y. Gorbunov, S. R. Klein, and J. Nystrand, “Two-Photon Interactions with Nuclear Breakup in Relativistic Heavy Ion Collisions”, Phys. Rev. C 80 (2009) 044902, doi:10.1103/PhysRevC.80.044902, arXiv:0907.1214.

[8] CMS-PAS-FSQ-13-006: https://cms-physics.web.cern.ch/cms-physics/public/FSQ-13-006-pas.pdf

[9] V. Andreev et al., "Performance studies of a full-length prototype for the CASTOR forward calorimeter at the CMS experiment”, Eur. Phys. J. C 67 (2010) 601, doi:10.1140/epjc/s10052-010-1316-4.

[10] R. Ciesielski and K. Goulianos, Proc. Sci., ICHEP (2013) 301 [arXiv:1205.1446].

[11] ATLAS Collaboration, "Rapidity gap cross sections measured with the ATLAS detector in pp collisions at $\sqrt{s}=7$ TeV”, Eur. Phys. J. C 72, 1926 (2012).

[12] CMS Collaboration, "Measurement of diffractive dissociation cross sections in $p p$ collisions at $\sqrt{s}=7$ TeV”, Phys.Rev. D 92, 012003 (2015) [arXiv:1503.08689v1] 